\title{
xADL: Enabling Architecture-Centric Tool Integration With XML
}

\author{
Rohit Khare $^{\dagger}$ \\ Nenad Medvivovic ${ }^{\text {t† }}$ \\ ${ }^{\dagger}$ Department of Information and Computer Science \\ University of California, Irvine \\ Irvine, CA 92697-3425, USA \\ \{rohit,mgunters,peymano,taylor\}@ics.uci.edu
}

Peyman Oreizy $^{\dagger}$

\author{
Richard N. Taylor ${ }^{\dagger}$ \\ ${ }^{\mathbf{t}}$ Computer Science Department \\ University of Southern California \\ Los Angeles, CA 90089-0781, USA \\ neno@usc.edu
}

\begin{abstract}
In order to support architecture-centric tool integration within the ArchStudio 2.0 Integrated Development Environment (IDE), we adopted Extensible Markup Language (XML) to represent the shared architecture-inprogress. Since ArchStudio is an architectural style-based development environment that incorporates an extensive number of tools, including commercial off-the-shelf products, we developed a new, vendor-neutral, ADL-neutral interchange format called Extensible Architecture Description Language (xADL), as well as a 'vocabulary' specific to the $\mathrm{C} 2$ style (xC2). This paper outlines our vision for representing architectures as hypertext, the design rationale behind $\mathrm{xADL}$ and $\mathrm{xC2}$, and summarizes our engineering experience with this strategy.
\end{abstract}

Keywords: Software environments, software architectures, off-the-shelf tool integration, XML

\section{INTRODUCTION}

The widespread adoption of Software Engineering Environments (SEE) predates the term itself. The rational decomposition of the development process and its artifacts can arguably be traced back to the advent of separate compilation. The zeroth generation of SEE integration took files as its primary unit of discourse between various tools. The well-known UNIX pipe-and-filter architectural style was entirely based on the concept that one tool's output data could be used as an input to another [11].

The first step, then, was a repository-centric approach for managing these artifacts. In such an Integrated Development Environment (IDE), different tools would work upon a central, shared database representing the product-inprogress. The archetype of this generation was the Stoneman reference model for the Ada Program Support Environment [7]. Interlisp [26] can be seen as one instance of this approach with suite of tools operating on a shared parse-tree.
A versioned filesystem was another popular variant, notably Revision Control System (RCS) [27].

Continuing the ascent, a second generation of processcentric IDEs emerged in the 1980s which took relations between these artifacts and their associated workflows as its unit of discourse. Tools such as Marvel [3] assisted developers by automating basic process steps and coordinating the work of tools "outside" the development path proper. In the extreme, IDE support tools maintained only those relations, as in the Chimera Linkbase [1].

Finally, we see the current era as the advent of architecturecentric IDEs that control the evolution of software throughout its lifecycle using architecture descriptions as its primary unit of discourse. As an example, ArchStudio 2.0 assumes the existence of versioned repositories and process automation in its foundation, and so focuses on the design, evaluation, instantiation, and editing of C2-style architectures. Supporting tool integration in this generation now requires an open, hypertext web representing the entire product, from architecture down to development artifacts.

We developed an Extensible Markup Language (XML) syntax for Architecture Description Languages (XADL) and customizations to the $\mathrm{C} 2$ style in particular $(\mathrm{xC} 2)$ in support of these goals. Furthermore, we integrated our XML Abstract Syntax Tree (AST) and an Abstract Data Type (ADT) representing the architecture-in-progress to the ArchStudio 2.0 environment on the fly, thereby highlighting the ease of dynamism in an IDE itself designed in the C2 style. By wrapping a parsed, shared representation of the work at hand behind a (potentially-distributed) event notification interface, our approach technologically updates the basic strategy of Field [23].

The balance of this paper focuses particularly on the role XML played in the integration of several tools within ArchStudio 2.0. We shall outline the origins and promise of XML broadly, our detailed design of $\mathrm{xADL}$ and $\mathrm{xC2}$, our implementation experience with it, and a discussion of its implications for broader and deeper tool integration within architecture-centric IDEs.

\section{EXTENSIBLE MARKUP LANGUAGE (XML)}

The HyperText Markup Language (HTML) allows the 
structural markup of World Wide Web documents. Now, HTML's evolutionary successor, XML, takes document markup to the next level, by offering human-readable semantic markup that is also machine-readable. As a result, XML makes it dramatically easier to develop and deploy new mission-specific markup, enabling the automation of the authoring, parsing, and processing of networked data.

Broadly speaking, the XML 1.0 standard [6] is a simplification of the Standard Generalized Markup Language (SGML) which itself dates back to the mid-60s. XML should be seen as a toolkit for creating new elements (also known as 'tags') and attributes upon them, as well as grammar rules governing the parse tree. All of these rules are captured in a Document Type Definition (DTD), which can be used to fomally validate any XML instance (file) against it.

More usefully, though, XML defines a lower level of conformance known as 'well-formed'. This level merely assures that all the elements open and close properly, and so on. Such purely mechanical checking allows designers to 'mix and match' elements from several DTDs. In particularly, the XML Namespaces facility allows us to interpret unknown element names as URLs which can be further investigated automatically or by hand. Namespaces thus disambiguate potentially conflicting tag semantics.

Our data integration strategy for ArchStudio 2.0 adopted XML for several of the properties introduced above:

- A text format governed by an open standard promised future-proof file formats. Furthermore, the advent of hybrid XHTML modules already provided a rich tagset for human-readable documentation and presentation of the architecture-in-progress.

- Well-formed XML accommodated multiple tools' own subtrees within the data model, as well as tool-specific attributes decorating existing elements.

- Namespaces explicitly articulated separate control over the vocabulary for describing high-level architectures in common (xADL), style-specific features (xC2), and toolspecific additonal data.

- Intrinsic support for hypertext linking encouraged future refactoring of architecture description into separate resources describing individual components and types, potentially published remotely by several developers (hyperlinked reuse).

- Finally, rich protocols for accessing, managing, and versioning XML repositories already existed, in the form of WebDAV (Web Distributed Authoring and Versioning) extensions to HTTP and the XPointer language for hyperlinking directly within XML documents

\section{APPROACH}

ArchStudio [20] is an architecture-centric IDE, based on the C2 architectural style [25]. The C2 style is an event- and component-based style, which allows dynamic evolution of the software system at runtime [12], due to its enforced decomposition into units of computation and data store, called components, and units that enable the interaction among components, called connectors, as suggested by David Garlan and Mary Shaw [23]. The highly dynamic nature of the $\mathrm{C} 2$ style is primarily caused by a central rule of the style, which does not allow direct links between two components, but rather requires the involvement of a connector in between. Hence components may be plugged in and out of the system without leaving another component behind with a dangling link. The separation of architectural units into components and connectors may be regarded analog to the nodes and transitions concept of Petri nets [21], where direct links may only exist from nodes to transitions and vice versa, but not within each domain. However, the $\mathrm{C} 2$ concept differs insofar as connector may be linked directly to other connectors.

ArchStudio not only supports the development of C2 style software, but was itself implemented in the C2 style. Such support for software evolution made ArchStudio itself an excellent target for incremental tool integration. Version 2.0 built upon a suite of tools already developed for ArchStudio 1.0 to add a new shared repository format (xADL), new style-checking tools, and several commercial- and researchgrade off-the-shelf tools, including Rational Rose [22], Armani [19], Metamata [14], and JavaBeans [24] as shown in Figure 1. 


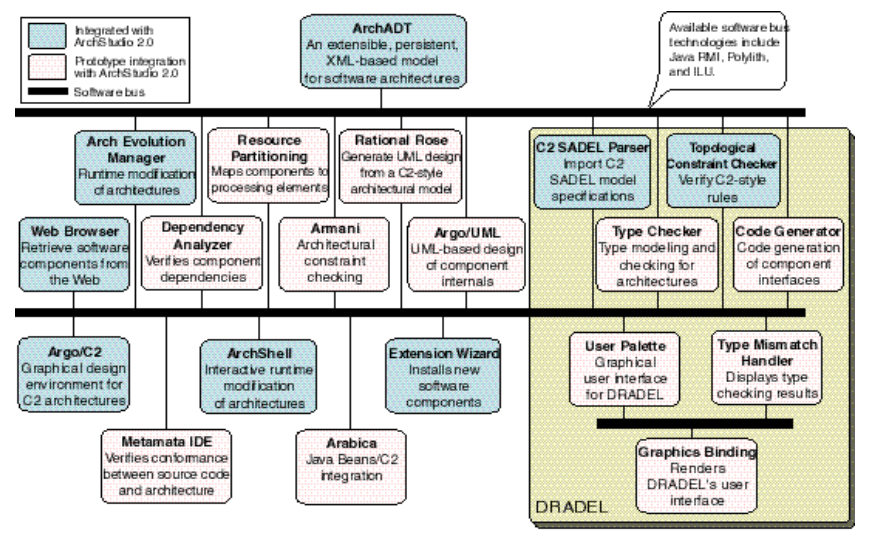

Figure 1. .ArchStudio 2.0 integrates several tools in the $\mathrm{C} 2$ style.

The first step required introducing an abstract data type representing the architecture-in-progress, ArchADT, quite apart from its new representation in an XML-based abstract syntax tree (AST). Each step had a beneficial consequence:

1. Since the message-based C2 style transmits by-copy rather than by-reference, globally sharing an ArchADT replaced the habit of sending enormous messages representing the entire architecture-in-progress. Furthermore, the new technique removed artificial sequence dependencies for tools that modified disjoint aspects of the architecture.

2. While ArchStudio 1.0 tool integration was either limited to tools that understood its internal application programming interface (API) or were mapped to it using component wrappers, the advent of XML at least eased data integration with off-the-shelf tools. Since the AST supported lowest-common-denominator access for tools which added new subtrees of information, or new attributes of existing objects, an XML AST enabled integration of tool-specific information transparently, within the ArchADT.

In particular, we designed xADL to be a shared language for representing a variety of possible ADLs. It introduces five basic tags, namely <Architecture>, <Component>, <Connector>, <ComponentType>, and <ConnectorType>, each with its own subtrees as well as hyperlinks between them.

- xADL

- Architecture

- Links

- Component

- Supports

- ComponentType

- Interface

- Parameter

- Connector

- Supports

- ConnectorType

- Interface

- Parameter

where

- $x A D L$->Architecture->Links specifies a list of directed hyperlinks between component and connector instances (links to <Component> and <Connector $>$ tags, respec- $<!--x C 2$ adds one parameter to the xADL:ConnectorType tag namely a message filtering policy with a default of none -->

$<$ !ELEMENT xADL:ConnectorType (Supports*)>

$<$ !ATTLIST xADL:ConnectorType name CDATA \#REQUIRED

\begin{tabular}{l|l}
$\times \mathrm{x} 2$ :filter & $\begin{array}{l}\text { (no filtering } \\
\text { notification filtering } \\
\text { message_filtering } \\
\text { prioritized } \\
\text { message_sink) }\end{array}$
\end{tabular}

Figure 2. An excerpt from the combined xADL and xC2 DTD tively),

- Component->Supports specifies name and type(s) supported by a component instance,

- ComponentType->Interface specifies name and method interfaces for each component type,

- ComponentType->Interface->Parameter specifies input and output parameters of a component interface,

- Connector->Supports specifies name and type(s) supported by a connector instance,

- ConnectorType->Interface specifies name and method interfaces for each connector type, and

- ConnectorType->Interface->Parameter specifies input and output parameters of the connector interface.

xADL may be extended to support a partciular architectural style, such as $\mathrm{C} 2$, by mixing-in additional XML Namespaces. In our case, $\mathrm{xC} 2$ added C2-specific tags, attributes, and constraints to the specification, as shown in the example in Figure 2.

$\mathrm{xADL}$ also supports the storage of tool-specific information as well-formed XML data, though at the expense of formal validation against a single, unified DTD. Leaving the interpretation of data to participating tools still allows ArchStudio to centrally manage and monitor the architecture-in-progress, even if it does not "understand" the data it stores.

\section{IMPLEMENTATION ISSUES}

The first tool to be upgraded was DRADEL (Development of Robust Architectures using a Description and Evolution Language) [18]. As the hub of the first-generation system, it was used to model architectures in the C2 style, check and enforce style constraints, manage heterogeneous subtyping, and generate application skeleton code. The central artifact representing the architecture-in-progress was a read-only, edit-externally C2SADEL text file.

Our new repository strategy was implemented at two layers: a 'physical' AST that guaranteed storage of well-formed XML fragments; and a 'logical' ArchADT that enforced the grammar and semantic rules (validity) of xADL and $\mathrm{xC} 2$. For the former service, we published a request-response message interface for basic tree manipulation: CreateTree, AddChildNode, DeleteNode, SetAttribute, GetNodelnfo, ReadFromFile, SaveToFile, and so on in the manner of Figure 1. At the logical layer, we preserved the existing interface at a higher level of abstraction, such as 
NewArchitecture, AddComponent, BasicSubtypeOf, and so on. Two functions were used throughout the class hierarchy to keep the two levels synchronized, namely toAST and fromAST; each logical object in the architecture maintained a 'shadow node' in the AST with its current state.

Thus, by contrast to the hand-coded text input parser of the previous generation, using off-the-shelf XML parsing technology automatically provided 'round-trip' input and output of the AST at any point.

To dynamically evolve from the existing ArchitectureSpec object that reflected a C2SADEL-format input file to the new ArchADT object bound to an XML-format representation, we applied a transition strategy which mirrored the notification stream used to construct the former onto the latter. The central challenge within that process, in turn, was managing the extreme parallelism of our new XML AST store, since the original environment was entirely sequential by virtue of passing the entire ArchitectureSpec object by value from parser to parser. Naturally, replacing massive copying of the entire architecture-in-progress with many more small queries against a shared representation eased physical distribution of ArchStudio 2.0 across LANs. Replacing in-process procedure calls with remote ones has its limits at Internet-scale, however. While we did not enact ArchStudio 2.0 across high-latency public Internet links, the 'chatty' pattern of AST access could accumulate into unacceptable overall performance.

Serializing edits to the shared AST was another difficult problem to address within the $\mathrm{C} 2$ event-based architectural style. Since it does not make any assumptions about the order messages are sent and retrieved within the system, edits must be tracked by unique identifiers or treated as idempotent (that is, reorderable). Of course, one atomicity solution is strict sequencing of requests and responses with one speaker at a time, as ArchStudio 1.0 did. This corresponds to a depth-first construction of the AST, while our parallel approach permits multiple tools to construct or analyze portions of the architecture simultaneously.

The problem, then, is that several messages or notifications of the exact same type, sometimes even with the same parameters, might be outstanding at the same time. Two notices that ChildAdded $38<$ Component> 40 and ChildAdded $38<$ Component> 41 cannot be reliably demultiplexed back to their respective initiators (the first numerical parameter is a common parent id, as shown in Figure 1, and the second is a newly-minted nodeID not initially known to the requestor).

Our solution was an additional optional parameter which identified the calling component, ref_com. Sending a pointer to the calling object on a round-trip through the AST interface was essential to decentralizing access to it within ArchStudio2 (i.e. multiple parallel readers and writers). Note, though, that the sequential delivery of requests by the C2 connector interface wrapping the AST still implicitly serialized all $\mathrm{I} / \mathrm{O}$ requests.

The complete decomposition of ArchADT includes several more classes, as shown in Figure 1. AST, ASTNode, and
ASTAttribute represent the tree anchor (or root), nodes within the tree, and attributes of the tree nodes, respectively. Good Java programming style packaged the constant string values and request names within an APIConstants class, as well as a central ASTExceptions class. Finally, the entire suite was encapsulated by an ASTComponent class which handled the external event interface according to the $\mathrm{C} 2$ style.

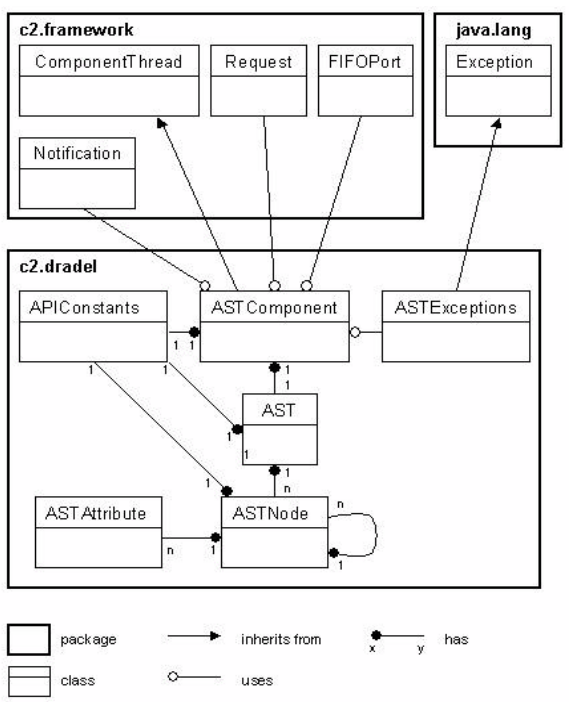

Figure 1. ArchADT component architecture in Booch notation [4].

\section{DISCUSSION}

There are several interesting implications of our adoption of $\mathrm{XML}$ and our design of $\mathrm{xADL}$ and $\mathrm{xC} 2$ in particular. First, adopting a standard syntax for representing the tool data being integrated within ArchStudio 2.0 should generate compelling 'network effects' for our product. That is to say, there is dramatic potential for reuse with XML over our previous custom text format. Already, we have been able to leverage off-the-shelf parsing technology, user interfaces (Figure 1), syntax-directed editors (Figure 2), and schema development tools.

Most of all, the XML developer community is already oriented towards enabling 'mix-and-match' reuse of application-specific ontologies. For example, the World Wide Web Consortium is developing the Scalable Vector Graphics language (SVG, [8]) for drawings. A future Unified Modeling Language (UML, [5]) graphical editor could produce SVG documents which could be transparently annotated with $\mathrm{xADL}$ and $\mathrm{xC} 2$ descriptions of the components and connectors those boxes and lines represent.

Second, the approach we have adopted in XADL can be easily extended to support multiple architecture description languages (ADLs), even within a single XML schema. Our extensive study of ADLs [16] has indicated that most all mainstream ADLs agree on the existence of components, connectors, and their configurations. A small number of ADLs, including Rapide [12] and Darwin [13], do not explicitly model connectors. However, even these ADLs 
support simple component interconnections; furthermore, Rapide employs specialized "connection components" to support more complex interactions. Additionally, all ADLs model component interfaces and do so in a relatively uniform fashion. Therefore, these shared aspects of ADLs would become part of the basic XADL schema.

That basic schema could then be extended in a number of ways to represent the varying parts of architectural descriptions across ADLs, such as the manner in which ADLs model architectural semantics, support evolution (both at system design time and run time), constrain the architecture (and its evolution), and so forth. Thus, for example, an xADL schema could simultaneously describe architectures specified in C2SADEL [18] and Wright [1]. If a particular tool is interested in the static model of behavior, it would access C2SADEL's component invariants and preand postconditions; alternately, if the tool is interested in the system's dynamic semantics, it would access Wright's CSPrelated items and ignore others. Another possibility that $\mathrm{xADL}$ affords us is the support for multiple configurations of the same set of components, where we access the part of the schema representing the specific configuration we are interested in, disregarding all other configurations.

Figure 1. An example xADL file viewed in Microsoft Internet Explorer 5.0 rendered by our default XSL stylesheet

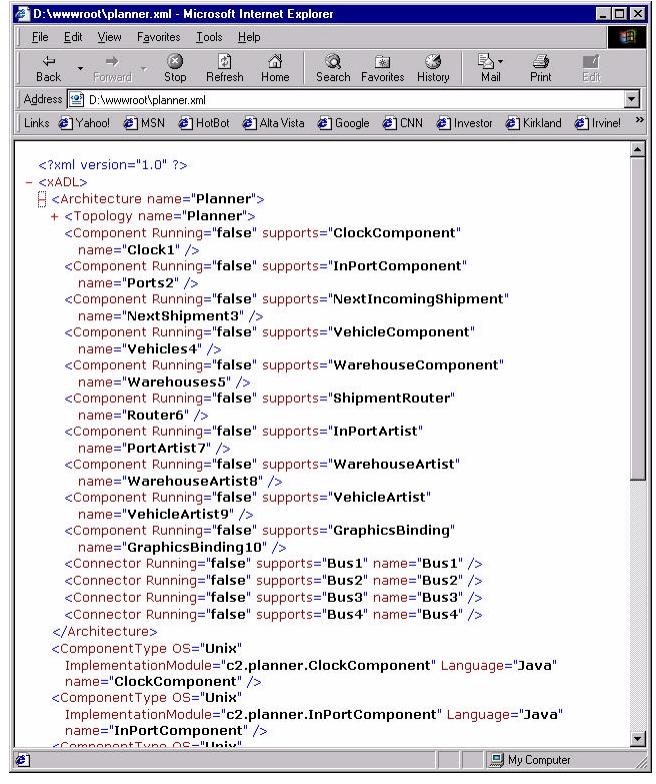

Figure 2. IBM's Xeena is a DTD-driven editor which can thus automatically enforce XML validation rules on $\mathrm{xADL} / \mathrm{xC} 2$ files

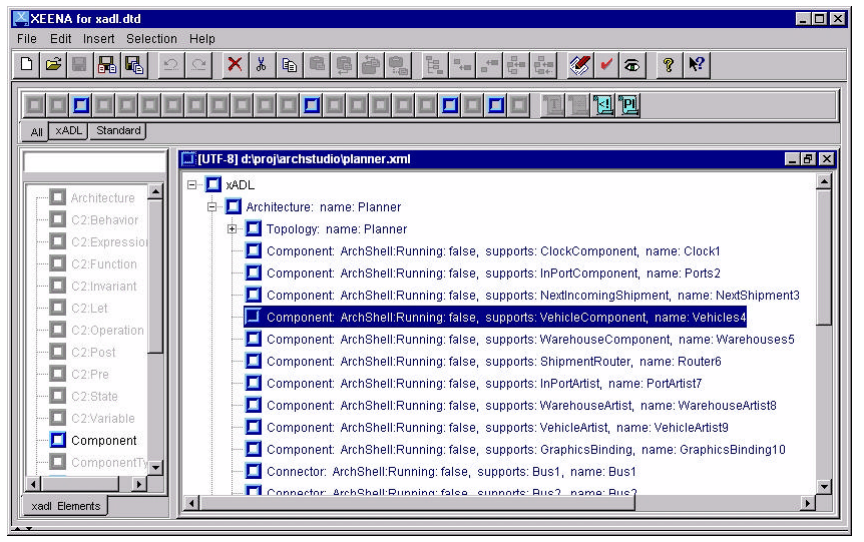

\section{CONCLUSIONS}

We adopted XML as a key technology for enabling architecture-centric tool integration in the ArchStudio 2.0 IDE. The C2 style eased the evolution from the previous version's custom text file format, C2SADEL, to a generic XML AST as the repository. This had immediate benefits for integrating several tools' data in the same file, for annotating existing data without interfering with its original use, and for hyperlinking to external data transparently.

Furthermore, we developed a new ontology for describing entire families of Architecture Description Languages (ADLs). By extracting the five most common abstractions and their relations into a top-level xADL namespace, we were able to separately represent data specific to the $\mathrm{C} 2$ architectural style and $\mathrm{C} 2 \mathrm{SADEL}$ in a subsidiary $\mathrm{xC2}$ namespace.

These technologies directly aided a strictly distributed team to integrate a substantial set of research and commercial tools within ArchStudio 2.0. Our eventual aim is even wider, to support Internet-scale development, with potentially large and varying developer communities composing systems over long times and distances [8]. Representing architectures as hypertext affords us reach; extracting our ontology in XML promises depth, through integration with generic, non-ADLaware XML applications.

\section{ACKNOWLEDGMENTS}

We wish to acknowledge the following individuals for their participation in the work described in this paper. ArchStudio 1.0 was developed by P. Oreizy and N. Medvidovic. ArchStudio 2.0 was developed by P. Oreizy, R. Khare, M. Guntersdorfer, K. Nies, E. Dashofy, Y. Kanomata, R. Natarajan, A. Hitomi, R. Klashner, L. Pan, M. Dias, M. Vieira, S. Devanathan, and J. Robbins.

This effort was sponsored by the Defense Advanced Research Projects Agency, and Air Force Research Laboratory, Air Force Material Command, USAF, under agreement numbers F30602-97-2-0021 and F30602-99-C0174. The U. S. Government is authorized to reproduce and distribute reprints for Governmental purposes notwithstanding any copyright annotation thereon. 


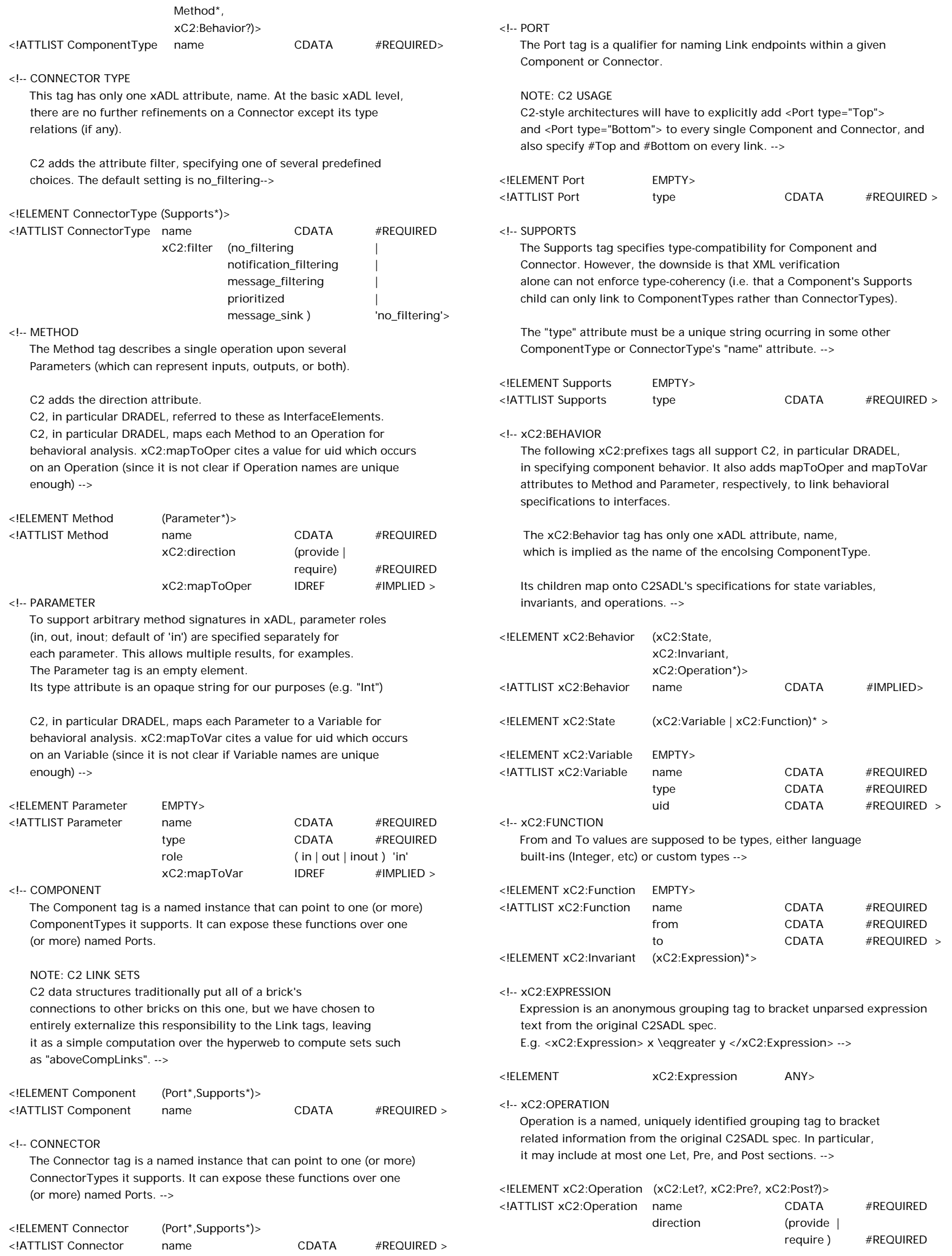


<EELEMENT xC2:Let

CDATA

\#REQUIRED >

$\angle$ EELEMENT $\times C 2$ :Pre $<$ EELEMENT xC2:Post

(xC2:Expression)> (xC2:Expression)> 